\title{
Anatomia do fígado de fetos de Guinea pig em final de gestação (Cavia porcellus [Linnaeus, 1758])
}

\author{
Mariangela de Toledo Barbino ${ }^{1}$ \\ Claudia Marinovic Oliveira ${ }^{2}$ \\ Erika Toledo Fonseca ${ }^{2}$ \\ Phelipe de Oliveira Favaron ${ }^{2}$ \\ Márcio Nogueira Rodrigues ${ }^{2}$ \\ Maria Angélica Miglino ${ }^{3}$ \\ ${ }^{1}$ Universidade Paulista, São José dos Campos - SP, Brasil \\ ${ }^{2}$ PPG em Anatomia dos Animais Domésticos e Silvestres, Departamento de Cirurgia \\ Faculdade de Medicina Veterinária e Zootecnia, Universidade de São Paulo \\ São Paulo - SP, Brasil \\ ${ }^{3}$ Departamento de Cirurgia, Faculdade de Medicina Veterinária e Zootecnia \\ Universidade de São Paulo, São Paulo - SP, Brasil \\ *Autor para correspondência \\ Rua Raul Cornélio Brom, 11, Nova Caçapava, CEP 12283-120, Caçapava - SP, Brasil \\ mari_barbino@yahoo.com.br
}

\section{Resumo}

Descrever a anatomia do fígado de fetos de Guinea pig (Cavia porcellus [Linnaeus, 1758]) em final de gestação e as caracterizações anatômicas, que possam fundamentar e auxiliar a compreensão da fisiologia da circulação fetal. Foram utilizados três fetos de Guinea pig em final de gestação, os quais foram dissecados e tiveram seus fígados retirados. Estes foram analisados, descritos e fotodocumentados macroscopicamente e microscopicamente por meio de microscopia de luz em coloração HE e microscopia eletrônica de varredura. Macroscopicamente, os fígados dos fetos possuem coloração castanha avermelhada e sua divisão em lobos é nitidamente observada, assim como a presença da vesícula biliar. O fígado divide-se em lobo lateral esquerdo, lobo medial esquerdo, lobo lateral direito, lobo medial direito, lobo quadrado e lobo caudado com processos papilar e caudado. Por meio da microscopia de luz, observa-se o tecido intensamente vascularizado, sendo que a porção esquerda do fígado recebe o sangue oriundo da placenta pela veia umbilical, e a porção direita é irrigada pela veia porta. As estruturas encontradas no fígado dos fetos de Guinea pig no final de gestação possuem semelhanças anatômicas aos de outras espécies de mamíferos.

Palavras-chave: Circulação, Fetos, Fígado, Guinea pig

\section{Abstract}

Anatomy of the liver of Guinea pig fetuses in late gestation (Cavia porcellus [Linnaeus, 1758]). To describe the anatomy of the liver of Guinea pig (Cavia porcellus [Linnaeus, 1758]) fetuses in late gestation and to obtain anatomical characteristics that can support and help the understanding of the physiology of fetal 
circulation. Three fetuses of Guinea pig in late gestation have been used, which were dissected and had their livers removed. These were analized, described, and photographed macroscopically and microscopically through light microscopy in HE and scanning electronic microscopy. Macroscopically, the fetuses livers have a reddish brown color and their division into lobes is clearly seen, as well as the presence of the gallbladder. The liver is divided into left lateral lobe, left medial lobe, right lateral lobe, right medial lobe, quadrate lobe, and caudate lobe with caudate and papillary processes. Through light microscopy, highly vascularized tissue is observed, and the left portion of the liver receive blood from the placenta through the umbilical vein, and the right portion is irrigated by the portal vein. The structures found on the liver of Guinea pig fetuses in late gestation are anatomically similar to those of other mammalian species.

Key words: Circulation, Fetuses, Guinea pig, Liver

\section{Introdução}

Muitos trabalhos vêm sendo realizados, principalmente em roedores, pois o conhecimento da fisiologia e morfologia da circulação fetal é importante para a compreensão de estudos farmacológicos e fisiológicos em humanos (LALEMAN et al., 2006). Kiserud e Acharya (2004) ressaltam que apesar de muitos estudos demonstrarem grande similaridade entre a circulação fetal e a de humanos, é importante conhecer a anatomia e fisiologia dos animais utilizados como modelo experimental para minimizar erros comparativos. Laleman et al. (2006) expõem ainda que, considerações éticas limitam procedimentos em seres humanos, sendo imprescindível a utilização de animais para o estudo de patologias hepáticas, para isso o conhecimento da anatomia e fisiologia do órgão em modelos experimentais devem ser bem conhecidos.

O fígado é o segundo maior órgão do corpo e a maior glândula. Está situado na cavidade abdominal abaixo do diafragma (JUNQUEIRA; CARNEIRO, 2008). Do ponto de vista funcional, é o local do organismo onde ocorrem numerosos processos metabólicos, pois ao receber a maior parte do material absorvido dos intestinos, metaboliza, armazena, sintetiza e elimina diversas substâncias, fatores que conferem a este órgão uma grande importância (BORGES et al., 2002).

Nos fetos, a veia umbilical penetra o fígado na fissura umbilical e emite ramos colaterais que vascularizam a porção esquerda do fígado, enquanto a veia porta vasculariza a porção direita (DYCE et al., 2010).

Em grande parte dos mamíferos um prolongamento do tronco umbilical, o ducto venoso, penetra através do parênquima do fígado, desviando da circulação hepática e unindo-se à veia cava caudal (DYCE et al., 2010). Detmer (1992) observou que em fetos de Guinea pig não há ducto venoso e todo retorno venoso umbilical atravessa o fígado.

Considerando-se a importância que este órgão representa na circulação sanguínea elaborou-se este trabalho visando à obtenção de caracterizações anatômicas que possam fundamentar e auxiliar na compreensão de estudos voltados à físiologia da circulação fetal.

\section{Materiais e Métodos}

Para a realização do estudo foram utilizadas três fêmeas da espécie Cavia porcellus em final de gestação oriundas da doação de um criatório particular.

Os procedimentos de eutanásia foram realizados no laboratório de Anatomia Animal da Faculdade de Medicina Veterinária e Zootecnia da Universidade de São Paulo de acordo com o protocolo n ${ }^{\circ}$ 1954/2010 certificado pela Comissão de Ética no uso de animais da FMVZ/USP.

Os animais foram posicionados em decúbito dorsal e feita a incisão na região pré-retro-umbilical sobre a linha Alba. Após a exposição do útero os fetos foram coletados, realizou-se a dissecação e retirada do fígado dos fetos.

Os materiais destinados à microscopia de luz foram fixados em formaldeído a 10\% em tampão fosfato (PBS), $0,1 \% \mathrm{pH} 7,4$. Em seguida o material foi desidratado em banhos de etanol em concentrações crescentes (de 50 a 
$100 \%$ ) e diafanizado em xilol, seguido pela inclusão em parafina. Foram realizados cortes consecutivos de $5 \mu \mathrm{m}$ de espessura, corados pelo método de hematoxilinaeosina e fotodocumentados.

Os materiais destinados à microscopia eletrônica de varredura foram imersos em solução fixadora de Glutaraldeído 2,5\%, montados em base de alumínio (stubs) com cola de carbono e em seguida cobertos com ouro (3min), sendo, então, analisados e fotografados no microscópio eletrônico de varredura LEO 435VP.

\section{Resultados}

A descrição macroscópica do fígado do feto de Guinea pig (Cavia porcellus) foi realizada com ênfase na forma, disposição e lobação.

O fígado apresenta cor castanha avermelhada. Sua estática na cavidade abdominal do feto ocorre pelos ligamentos triangulares, ligamentos coronários, ligamento falciforme e pela veia umbilical intraabdominal, a qual após o rompimento do cordão umbilical dá origem ao ligamento redondo do fígado.

Sua morfologia permitiu identificar na superfície desse órgão as bordas, direita, esquerda, dorsal e ventral. A face parietal convexa, adaptada ao diafragma, e a face visceral côncava, com impressões dos órgãos vizinhos.

A divisão em lobos foi nitidamente observada, assim como a presença da vesícula biliar. As Figuras 1A, 1B, 1C, 1D mostram os lobos lateral esquerdo, medial esquerdo, lateral direito, medial direito, quadrado e caudado com os processos papilar e caudado.

O lobo lateral esquerdo possui espessura mais delgada e em sua face ventral estava uma pequena dilatação denominada língua do lobo esquerdo (Figura $1 \mathrm{~A}$ e $1 \mathrm{~B})$.

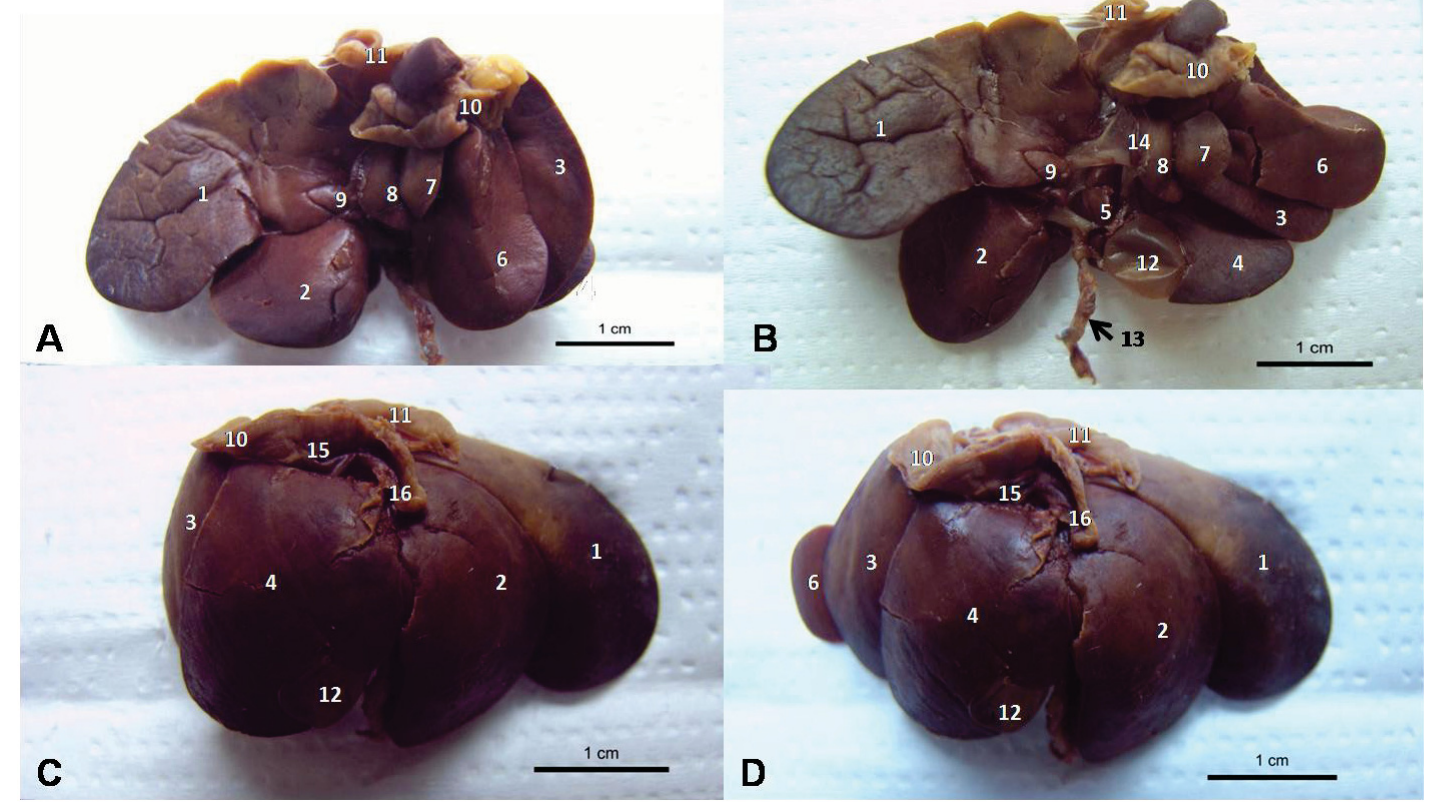

FIGURA 1: Fotografias macroscópicas do fígado do feto de Cavia porcellus. A e B: Face visceral. C e D: Face parietal. Estão enumerados: 1 - lobus sinister lateralis - lobo lateral esquerdo; 2 - lobus sinister medialis - lobo medial esquerdo; 3 - lobus dexter lateralis - lobo lateral direito; 4 - lobus dexter medialis - lobo medial direito; 5 - lobus quadratus - lobo quadrado; 6 - processus caudatus processo caudado do lobo caudado; 7 - processus papillaris, pars retroventricularis - processo papilar, retroventricular; 8 - processus papillaris, pars preventricularis - processo papilar, preventricular; 9 - lingula lobi sinistri - língua do lobo esquerdo; 10 - lig. triangulare dextrum - ligamento triangular direito; 11 - lig. triangulare sinistrum - ligamento triangular esquerdo; 12 - vesica fellea - vesícula biliar; 13 - veia umbilical; 14 - impressio esophagea - impressão do esôfago; 15 - lig. coronarium dextrum - ligamento coronário direito; 16 - lig. falciforme hepatis - ligamento falciforme. 
Entre o lobo medial esquerdo e o lobo medial direito se observa uma fissura por onde passa a veia umbilical intra-abdominal (Figura 1A e 1B).

Na porção cranial do processo papilar há uma incisura para o esôfago (Figura 1B).

Entre os lobos quadrado e medial direito, está a vesícula biliar fixada no lobo medial direito, encaixada profundamente, até atingir a superfície parietal, em contato com o diafragma (Figuras 1B, 1C e 1D).

O processo caudado do lobo caudado, juntamente com o lobo lateral direito, delimita a fossa renal, configurando a sintopia desse órgão com o rim direito (Figura 1A).

Os ligamentos triangulares direito e esquerdo, coronário direito e esquerdo e falciforme fixam o fígado firmemente à cavidade abdominal (Figura $1 \mathrm{C}$ e 1D).

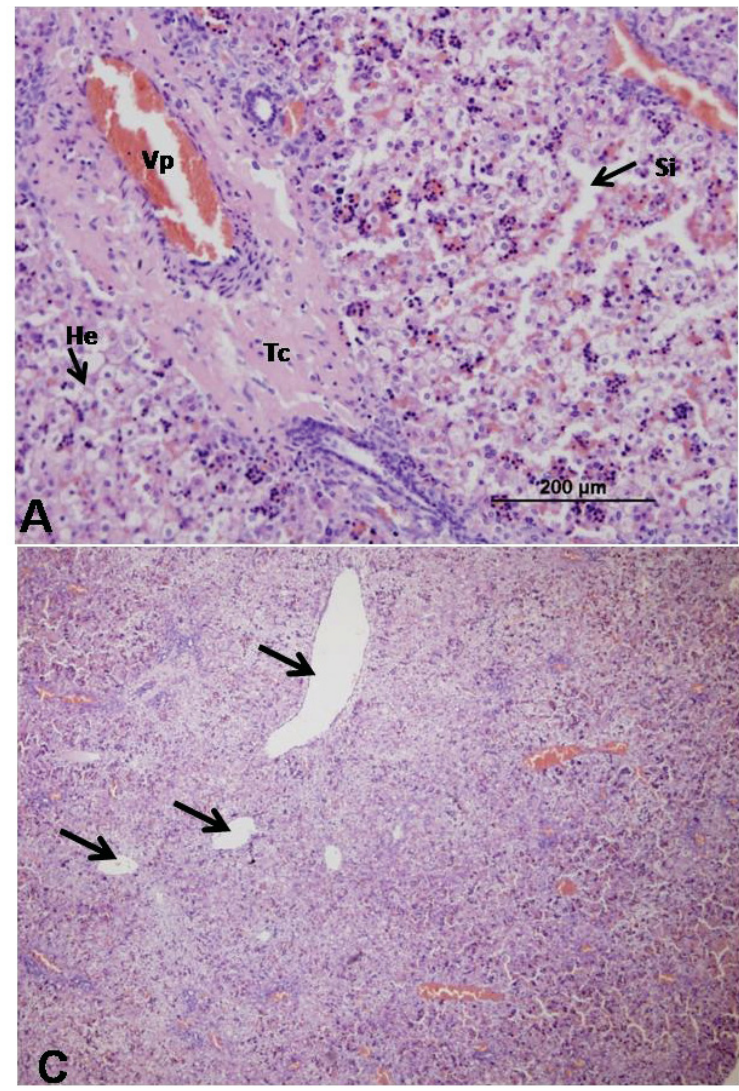

O tecido hepático está intensamente irrigado, demonstrando o grande papel do órgão no sistema circulatório (Figura 2A, 2B, 2C e 2D). Parte do suporte sanguíneo no fígado do feto é oriundo da placenta pela veia umbilical, que vasculariza a porção esquerda ramificando e vascularizando todo o tecido do órgão (Figura 2C). A porção direita é irrigada pela veia porta que também ramifica até a periferia dos lobos atingindo os capilares sinusóides. A partir dos capilares sinusóides se originam as veias centrais que gradualmente aumentam em diâmetro, convergem e fundem-se e desembocam na veia cava caudal.

A divisão do fígado em lobos ficou mais evidente na microscopia eletrônica de varredura (Figura 3A; 3B).

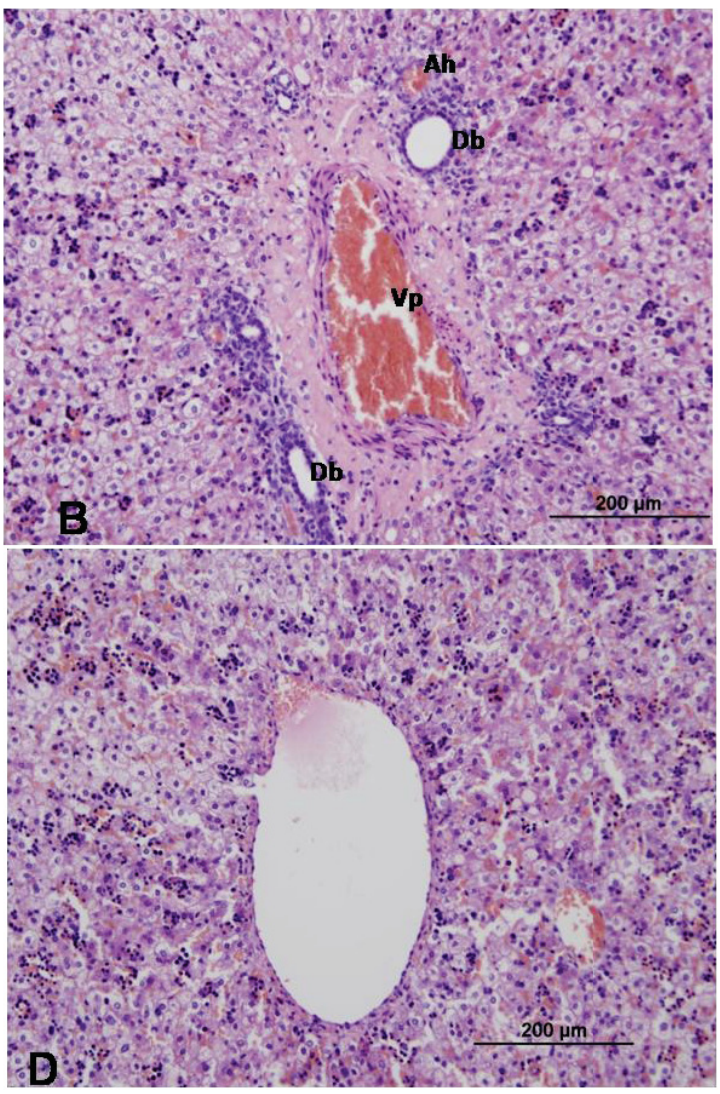

FIGURA 2: Fotomicrografias do fígado de feto de Cavia porcellus. A: Veia porta (Vp); Capilares sinusóides (Si); Tecido Conjuntivo (Tc) ao redor do ramo da veia porta, e a formação de cordões de hepatócitos $(\mathrm{He})$. Coloração hematoxilina e eosina, aumento 20x. B: Espaço porta contendo ramo da artéria hepática (Ah), ramo da veia porta (Vp), e ductos biliares ( $\mathrm{Db}$ ), observar o tecido conjuntivo circundando esses elementos do espaço porta. Coloração hematoxilina e eosina, aumento 20x. C:Veias centrais indicadas pela seta. Observar que o parênquima hepático não é subdividido em lóbulos. Coloração hematoxilina e eosina, aumento 4x. D: Veia central vista em maior aumento. Coloração hematoxilina e eosina, aumento 20x. 

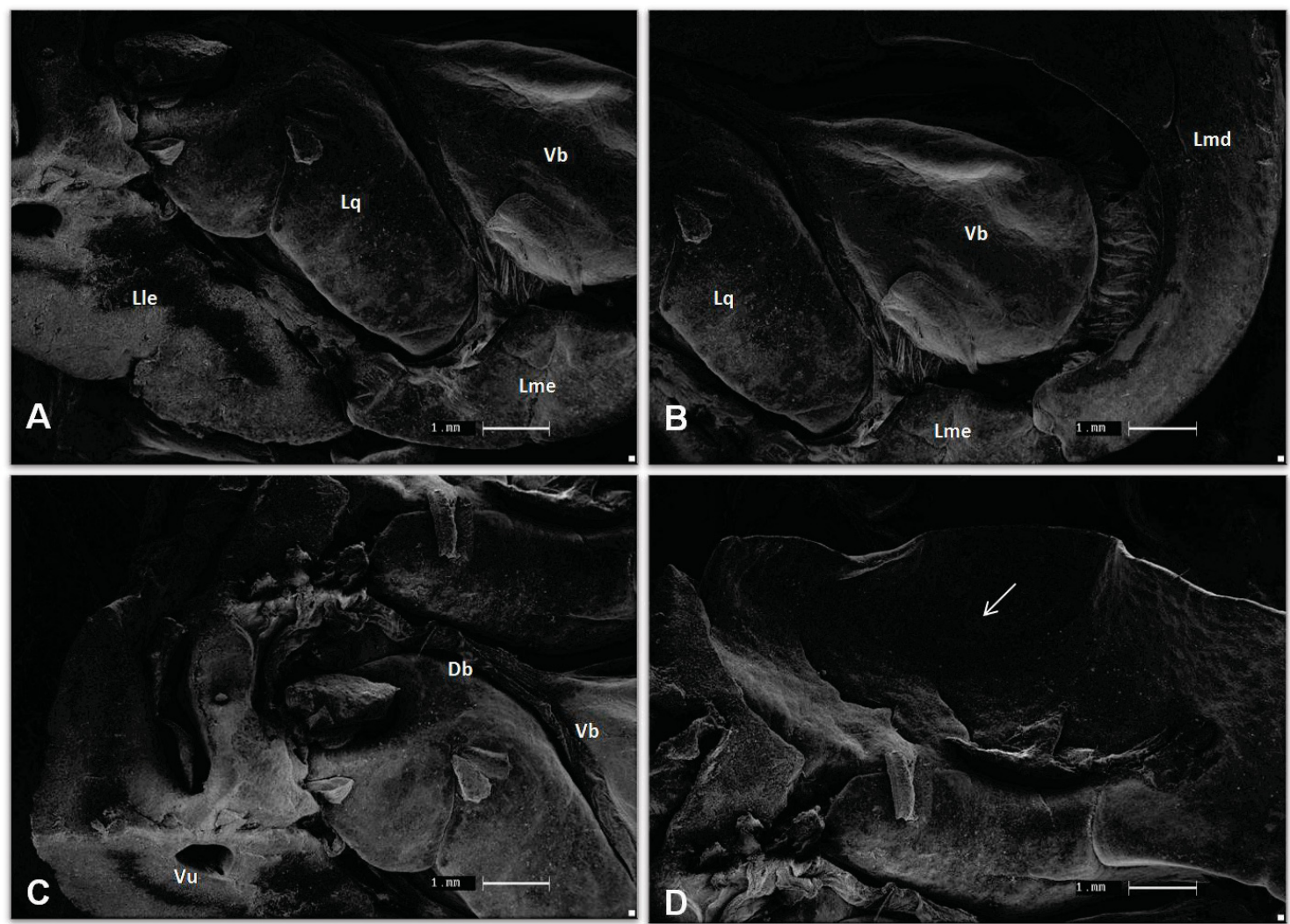

FIGURA 3. Fotomicrografias eletrônicas de varredura do fígado de feto de Cavia porcellus. A: Localização da vesícula biliar $(\mathrm{Vb})$ em relação ao lobo lateral esquerdo (Lle), lobo quadrado (Lq) e lobo medial esquerdo (Lme). B: Inserção da vesícula biliar ( $\mathrm{Vb}$ ) no lobo medial direito (Lmd); lobo quadrado (Lq) e lobo medial esquerdo (Lme). C: Partindo da vesícula biliar (Vb) observa-se o ducto biliar ( $\mathrm{Db})$. Também é possível visualizar o corte da veia umbilical (Vu). D: Impressão renal (seta) presente no processo caudado do lobo caudado.

\section{Discussão}

No presente estudo, a face parietal convexa, adaptada ao diafragma, e a face côncava, com impressões dos órgãos vizinhos encontradas em Guinea pig foram igualmente observadas em cervídeos por Borges et al. (2002), suínos e cães por Alves et al. (2007) e em avestruzes por Abidu-Figueiredo et al (2006).

Macroscopicamente observa-se que o fígado do feto de Guinea pig apresenta cor castanha avermelhada, em concordância com os dados de carnívoros descritos por Dyce et al. (2010); sua estática na cavidade abdominal deve-se aos ligamentos triangulares, ligamentos coronários, ligamento falciforme e pela veia umbilical intra-abdominal. A divisão em 6 lobos: lateral esquerdo, medial esquerdo, lateral direito, medial direito, quadrado e caudado; sendo o lobo caudado constituído pelos processos papilar e caudato morfologia esta que também foi observada em chinchilas, capivaras, suínos e carnívoros (PEREZ; LIMA, 2007; SOUZA et al., 2007; CASTRO et al., 2010; DYCE et al., 2010). Em contrapartida, estes dados diferem dos descritos em muçuãs por Machado Junior et al. (2005), que apresentam a morfologia externa semelhante ao dos principais animais domésticos, porém não apresentam processo caudado no lobo caudado.

A vesícula biliar, entre os lobos quadrado e medial direito, fixada no lobo medial direito, se encaixa profundamente, atingindo a superfície parietal, entrando em contato com o diafragma, como o descrito em gambás, por Koch et al. (1990), em capivaras, por Souza et al. (2007), e em chinchilas, por Castro et al. (2010). Estes dados são semelhantes à descrição de Junqueira e Carneiro (2008), que se refere à vesícula biliar como um órgão oco e em formato de pêra. Já em 
veados (Manzana americana, Blastocerus bezoarticus, Manzana simplicicornis e Blastocerus dichotomus) Miglino et al. (1993) e Borges et al. (2002) relataram a ausência da vesícula biliar.

Assim como no fígado de humanos, não foi possível delimitar em lóbulos o fígado do feto de Guinea pig, diferentemente dos suínos, onde Junqueira e Carneiro (2008) descrevem a presença de uma camada de tecido conjuntivo separando os lóbulos.

O lobo lateral esquerdo possui espessura mais delgada, e em face ventral, há uma pequena dilatação denominada língua do lobo esquerdo de acordo com as descrições feitas por Popesko et al. (1992).

Entre o lobo medial esquerdo e o lobo medial direito existe uma fissura por onde passa a veia umbilical intra-abdominal, semelhante ao demonstrado por Popesko et al. (1992).

Na porção cranial do processo papilar existe uma incisura para o esôfago, onde o esôfago, e o processo caudado do lobo caudado, juntamente com o lobo lateral direito, delimitam a fossa renal, configurando a sintopia desse órgão com o rim direito. Estes dados anatômicos também foram encontrados no cervo do Pantanal por Borges et al (2002). Em cães, Castro-Silva Jr. et al. (2002) relataram que os lobos hepáticos lateral direito e caudado são atravessados pela veia cava inferior.

Microscopicamente observou-se que o tecido hepático é intensamente irrigado, demonstrando o grande papel do órgão no sistema circulatório. De acordo com os achados de Carter et al (1992), essa irrigação deve-se em parte ao suporte sanguíneo oriundo da placenta através da veia umbilical, que vasculariza a porção esquerda do fígado, ramificando-se e vascularizando todo o tecido. Por outro lado, a porção direita que é irrigada pela veia porta também se ramifica até a periferia dos lobos atingindo os capilares sinusóides. Segundo Junqueira e Carneiro (2008), a partir dos capilares sinusóides terão origem as veias centrais que gradualmente aumentarão em diâmetro, convergindo e fundindo-se para desembocar na veia cava caudal. Em búfalos e macaco-prego a veia cava caudal foi visualizada próximo ao diafragma para receber as veias hepáticas (ALMEIDA; PRADA, 2001; ALVES et al., 2007).
Carter et al. (1992), estudando a circulação em fetos de Guinea pig, afirmaram que a distribuição das veias umbilical e porta determina o fluxo e o gradiente de pressão da circulação hepática no feto, constatando ainda que as áreas irrigadas por essas veias eram claramente delimitadas e não havia nenhuma válvula que pudesse explicar essa delimitação tão consistente concluindo que, um alto fluxo na veia umbilical é suficiente para evitar que o fluxo venoso portal atravesse para o lado esquerdo do fígado.

A partir dos resultados deste trabalho e das referências citadas pode-se concluir que o fígado dos fetos de Guinea pig são muito semelhantes ao de outras espécies. Essas informações auxiliarão na interpretação de estudos que utilizem a espécie Cavia porcellus como modelo de experimentação animal.

\section{Agradecimentos}

Á Fundação de Amparo à Pesquisa do Estado de São Paulo (FAPESP) pelo financiamento deste trabalho (Proc. 2010/08850-5) através da concessão de uma bolsa de Iniciação Científica.

\section{Referências}

ABIDU-FIGUEIREDO, M.; XAVIER-SILVA, B.; BATH, F. V. C.; BABINSKI, M. A.; CHAGAS, M. A. Aspectos morfológicos e topográficos do fígado de avestruz (Struchio camelus). Revista Portuguesa de Ciências Veterinárias, Lisboa, v. 101, n. 557-558, p. 41-43, 2006.

ALMEIDA, A. E. F. S.; PRADA, I. L. S. Comportamento anatômico da veia hepática esquerda de fetos de búfalos (Bubalus bubalis - Linnaeus, 1758). Revista Brasileira de Saúde e Produção Animal, Salvador, v. 1, p. 61-67, 2001.

ALVES, F. R.; COSTA, F. B.; AROUCHE, M. M. S.; BARROS, A. C. E.; MIGLINO, M. A.; VULCANO, L. C.; GUERRA, P. C. Avaliação ultrasonográfica do sistema urinário, fígado e útero do macaco-prego, Cebus apella. Pesquisa Veterinária Brasileira, Seropédica, v. 27, n. 9, p. 377-382, 2007.

BORGES, E. M.; MACHADO, M. R. F.; OLIVEIRA, F. S.; SOUZA, W. M.; DUARTE, J. M. B. Aspectos morfológicos do fígado do cervo do pantanal (Blastocerus dichotomus). Brazilian Journal of Veterinary Research and Animal Science, São Paulo, v. 39, n. 2, p. 78-80, 2002.

CARTER, A. M.; DETMER, A.; EGUND, N. Contribution of the umbilical and portal veins to the hepatic blood supply of guinea pig fetuses - an angiographic study. Laboratory Animal Science, Cordova, v. 42, n. 2, p. 174-179, 1992. 
CASTRO, T. F., DUMMER, R. J., RICKES, E. M., PEREIRA, M A. M. Aspectos morfológicos, morfométricos e topográficos do aparelho digestório de Chinchilla lanígera. Brazilian Journal of Veterinary Research and Animal Science, São Paulo, v. 47, n. 1 , p. 86-94, 2010.

CASTRO-SILVA JR., O.; RAMALHO, F. S.; PACHECO, E. G. Hepatectomia total com preservação da veia cava inferior: "piggyback" experimental em cães. Acta Cirúrgica Brasileira, São Paulo, v. 17, n. 3, p. 79-82, 2002.

DETMER, A. Intrauterine growth retardations. An experimental study of fetal growth, regional blood flow and hepatic lipid metabolism in the anesthetized guinea pig. 1992. $100 \mathrm{f}$. Tese (Akademisk Avhandling) - Sveiges Lantbruksuniversitet, Uppsala. 1992

DYCE, K. M.; SACK, W. O.; WENSING, C. J. G. Tratado de anatomia veterinária. 4 ed. Rio de Janeiro: Elsevier, 2010. 834 p. JUNQUEIRA, L. C.; CARNEIRO, J. Histologia básica. 11 ed. Rio de Janeiro: Guanabara Koogan, 2008. 524 p.

KISERUD, T.; ACHARYA, G. The fetal circulation. Prenatal Diagnosis, Bergen, v. 24, n. 13, p. 1049-1059, 2004.

KOCH, R.; GASSE, H.; WILKENS, H. Topographic anatomy of the abdominal organs of the gray short-tailed opossum, Monodelphisdomestica (Marsupialia). Zeitschrift für Versuchstierkude, Jena, v. 33, p. 251-258, 1990.
LALEMAN, W.; ELST, I.; ZEEGERS, M.; SERVAES, R.; LIBBRECTH, L.; ROSKAMS, FEVERY, J.; NEVENS, F. A stable model of cirrhotic portal hypertension in the rat: Thiocetamide revisited. European Journal of Clinical Investigation, London, v. 36, n. 4, p. 242-249, 2006.

MACHADO JUNIOR, A. A. N; SOUSA, A. L.; CARVALHO, F. C. F.; ALVES, F. R. Anatomia do fígado e vias bilíferas do muçuã (Kinosternon scorpioides). Archives of Veterinary Science, Curitiba, v. 10, n. 2, 125-133, 2005.

MIGLINO, M. A.; SOUZA, W. M.; DIDIO, L. J. A. Sistema excretor do fígado de veados (Manzana americana, Blastocerus bezoarticus e Manzana simplicicornis). Brazilian Journal of Veterinary Research and Animal Science, São Paulo, v. 33, n. 1, p. 19-23, 1993.

PEREZ, W.; LIMA, M. Anatomical descriptionof the liver, hepatic ligaments and omenta in the coypu (Myocastor coypus). International Journal Morphology, Temuco, v. 25, n. 1, p. 61-64, 2007.

POPESKO, P.; RAJTOVA, V.; HORÁK, J. A colour atlas of anatomy of small laboratory animals. v. 2. London: Wolfe, 1992. $255 \mathrm{p}$.

SOUZA, W. M.; SOUZA, N. T. M.; CARVALHO, R. G.; CORREA, C. N. Topografia da artéria hepática no fígado da capivara (Hydrochaerus hydrochaeris). Ciência Rural, Santa Maria, v. 37, n. 1, p. 141-145, 2007. 